\title{
THE LEVI FORM AND LOCAL COMPLEX FOLIATIONS
}

\author{
MICHAEL FREEMAN ${ }^{1}$
}

\begin{abstract}
A short coordinate-free proof is given for some known results on the existence of local complex-analytic foliations of a real submanifold $M$ of $C^{n}$. The proof uses an explicit formulation of the equivalence between two definitions of the E. E. Levi form of $M$.
\end{abstract}

A local definition will suffice for the submanifold; $M=\{z \in U: \rho(z)$ $=0\}$, where $\rho=\left(\rho_{1}, \ldots, \rho_{m}\right): U \rightarrow \mathbf{R}^{m}$ is part of a smooth real coordinate patch for $U$ open in $\mathbf{C}^{n}$. $C$ is the ring of smooth complex-valued functions on $U$ and $T \mathbf{C}^{n}$ the $\mathcal{C}$-module of smooth vector fields on $U$. Geometric objects on $M$ are regarded as residue classes $X^{\prime}=X+O(M)$ of objects $X$ on $U$, where $O(M)$ is the subspace of objects vanishing on $M$. Thus the ring $C^{\prime}$ of smooth functions on $M$ is $e^{\prime}=\mathcal{C} / O(M)$ and the $e^{\prime}$-module of smooth vector fields on $M$ is $T^{\prime}=T / O(M)$, where $T=\left\{X \in T C^{n}: X \rho_{j}=d \rho_{j}(X) \in\right.$ $O(M)$, all $j\}$. The submodule of complex tangent vector fields is $H^{\prime}=$ $H / O(M)$, where $H=\left\{X \in T \mathbf{C}^{n}: \partial \rho_{j}(X)\right.$ and $\bar{\partial} \rho_{j}(X) \in O(M)$, all $\left.j\right\}$.

The E. E. Levi form of $M$ is a bilinear map of $H^{\prime} \times H^{\prime}$ which can be conceived in (at least) two ways:

(1) as $L \rho: H^{\prime} \times H^{\prime} \rightarrow \mathcal{C}^{\prime m}$ defined by

$$
L \rho\left(X^{\prime}, Y^{\prime}\right)=\partial \bar{\partial} \rho(X, Y)^{\prime}=\left(\partial \bar{\partial} \rho_{1}(X, Y)^{\prime}, \ldots, \partial \bar{\partial} \rho_{m}(X, Y)^{\prime}\right),
$$

or

(2) as $L: H^{\prime} \times H^{\prime} \rightarrow T^{\prime} / H^{\prime}$ defined by $L\left(X^{\prime}, Y^{\prime}\right)=\left[X^{\prime}, Y^{\prime}\right]+H^{\prime}$, where $\left[X^{\prime}, Y^{\prime}\right]=[X, Y]^{\prime}$ for $X^{\prime}, Y^{\prime} \in T^{\prime}[5]$.

That (1) and (2) are essentially equivalent has been known for some time. Recently, an explicit coordinate-free expression of their relationship was suggested in [1, proof of Proposition 3.2.1]. Due to its usefulness, this fundamental fact deserves more emphasis:

(3) The differential $\bar{\partial} \rho$ induces a $\mathcal{C}^{\prime}$-monomorphism $\alpha: T^{\prime} / H^{\prime} \rightarrow \mathcal{C}^{\prime m}$ such that $L \rho\left(X^{\prime}, Y^{\prime}\right)=\alpha L\left(X^{\prime}, Y^{\prime}\right), X^{\prime}, Y^{\prime} \in H^{\prime}$.

This is proved by applying the standard identity $d \omega(X, Y)=X \omega(Y)-$ $Y \omega(X)-\omega([X, Y])$ to $\omega=\bar{\partial} \rho$ and $X, Y \in H$. It is easy to see that the first two terms on the right vanish on $M$ and conclude that

(4) $\partial \bar{\partial} \rho(X, Y)^{\prime}=-\bar{\partial} \rho([X, Y])^{\prime}, X, Y \in H$ (recall that $d \bar{\partial} \rho_{j}=\partial \bar{\partial} \rho_{j}$ ). Relation (4) says that the top triangle of the diagram below commutes.

Received by the editors July 25, 1975 and, in revised form, September 16, 1975.

AMS (MOS) subject classifications (1970). Primary 32C10, 53B25.

Key words and phrases. Levi form, complex tangent, local complex foliation.

${ }^{1}$ Research supported by NSF GP-43247 at the University of Kentucky.

c American Mathematical Society 1976 


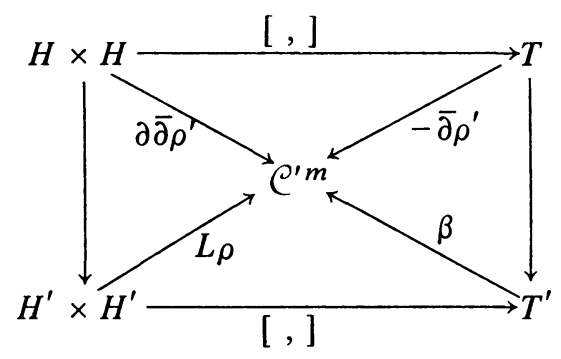

The vertical maps are natural projections and $\beta\left(X^{\prime}\right)=-\bar{\partial} \rho(X)^{\prime}$. Since the right and left triangles and the outer square commute, a simple chase of this diagram shows

(5) $L \rho\left(X^{\prime}, Y^{\prime}\right)=\beta\left(\left[X^{\prime}, Y^{\prime}\right]\right), X^{\prime}, Y^{\prime} \in H^{\prime}$.

Now $\operatorname{ker}\left(\beta \mid T^{\prime}\right)=H^{\prime}$ because $d \rho=\partial \rho+\bar{\partial} \rho$. Therefore there exists a unique monomorphism $\alpha: T^{\prime} / H^{\prime} \rightarrow \bigodot^{\prime m}$ such that $\alpha \circ \pi=\beta$, where $\pi$ : $T^{\prime} \rightarrow T^{\prime} / H^{\prime}$ is the quotient map. This and (5) show that $\alpha$ satisfies (3).

Relation (3) permits an easy proof of the existence of local complex foliations of $M$. Consider the null space $N^{\prime}=\left\{X^{\prime} \in H^{\prime}: L \rho\left(X^{\prime}, Y^{\prime}\right)=0\right.$ $\left.\forall Y^{\prime} \in H^{\prime}\right\}$, which is equivalent to the Levi null space defined pointwise in [2, Definition 2.7] under the constant rank assumptions made there. It requires a lot of calculation in [2] to prove (under these assumptions), a result [2, Theorem 6.1C] equivalent to the integrability condition $\left[N^{\prime}, N^{\prime}\right] \subset N^{\prime}$, yielding a foliation by complex submanifolds tangent to $N^{\prime}$. This integrability condition can be obtained directly by using (3) to rewrite $N^{\prime}$ as $N^{\prime}=\left\{X^{\prime} \in\right.$ $H^{\prime}:\left[X^{\prime}, Y^{\prime}\right] \in H^{\prime}$ for every $\left.Y^{\prime} \in H^{\prime}\right\}$ and then simply inspecting the Jacobi identity $\left[\left[X^{\prime}, Y^{\prime}\right], Z^{\prime}\right]+\left[\left[Z^{\prime}, X^{\prime}\right], Y^{\prime}\right]+\left[\left[Y^{\prime}, Z^{\prime}\right], X^{\prime}\right]=0$. It is clear that the Levi flat case $N=H$ is described by the equivalent conditions $L \rho=0$ and [ $\left.H^{\prime}, H^{\prime}\right] \subset H^{\prime}$, due to Sommer [4] when $M$ is a hypersurface. His proof and a later one in [3] require considerable calculation in coordinates.

\section{REFERENCES}

1. G. B. Folland and J. J. Kohn, The Neumann problem for the Cauchy-Riemann complex, Ann. of Math. Studies, no. 75, Princeton Univ. Press, Princeton, N. J., 1972.

2. M. Freeman, Local complex foliation of real submanifolds, Math. Ann. 209 (1974), 1-30. MR 49 \# 10911.

3. P. Kraut, $Z u$ einem Satz von $F$. Sommer über eine komplex-analytische Blätterung reeller

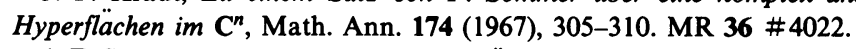

4. F. Sommer, Komplex-analytische Blätterung reeller Mannigfaltigkeiten im $\mathbf{C}^{n}$, Math. Ann. 136 (1958), 111-133. MR 21 \#730.

5. R. O. Wells, Jr., Function theory on differentiable manifolds, Contributions to Analysis, Academic Press, New York, 1974, pp. 407-441.

Department of Mathematics, University of Kentucky, Lexington, Kentucky 40506 